\title{
Long-Term Survival after Hospitalization for Community-Acquired and Healthcare-Associated Pneumonia
}

\author{
Laura M. Cecere ${ }^{a}$ Gordon D. Rubenfeld ${ }^{a, c}$ David R. Park ${ }^{a}$ Richard K. Root ${ }^{b, \dagger}$ \\ Christopher H. Goss ${ }^{a}$ \\ Divisions of a Pulmonary and Critical Care Medicine and ${ }^{\mathrm{b}}$ Infectious Diseases, Department of Medicine, University \\ of Washington School of Medicine, Seattle, Wash., USA; ' $D$ Division of Pulmonary and Critical Care Medicine, \\ Department of Medicine, University of Toronto, Toronto, Ont., Canada
}

\section{For editorial comment see p. 89}

\section{Key Words}

Community-acquired pneumonia $\cdot$ Epidemiology $\cdot$

Healthcare associated pneumonia - Outcomes •

Pneumonia

\begin{abstract}
Background: Elderly patients surviving community-acquired pneumonia (CAP) have subsequent increased mortality. However, little is known regarding long-term survival in younger adults or those with healthcare-associated pneumonia (HCAP). Objectives: To identify factors associated with mortality and compare long-term survival in patients hospitalized with HCAP to that of patients with CAP. Methods: We determined survival after discharge as of December 2002 in a patient cohort admitted with pneumonia between June 1994 and May 1996. We used the Cox proportional hazard model to estimate differences in survival after controlling for confounders. Results: Of the 522 patients hospitalized with pneumonia, 457 survived to discharge. One hundred sixty-four patients (36\%, 95\% confidence interval, $\mathrm{Cl}$, $31-40 \%)$ were admitted with HCAP, while 293 (64\%, 95\% Cl 60-69\%) were admitted with CAP. Of the 181 deaths in the follow-up period, 70 occurred in patients under age 65 years
\end{abstract}

\section{KARGER}

Fax +41613061234 E-Mail karger@karger.ch www.karger.com
() 2009 S. Karger AG, Basel

www.karger.com/res admitted with HCAP (53\% death rate, 95\% Cl 44-62\%). Nineteen of these deaths $(27 \%, 95 \% \mathrm{Cl} 17-39 \%)$ occurred in the absence of HIV infection. In patients under the age of 65 whose only risk factor for HCAP was treatment for pneumonia or hospitalization in the previous 90 days, 4 of 13 patients (31\%, 95\% Cl 9-61\%) died. Twenty percent (95\% Cl 15-26\%) of patients under age 65 years admitted with CAP died during the follow-up. Conclusions: Admission for HCAP, and to a lesser degree CAP, is associated with increased long-term mortality even in young patients. Future studies are warranted to identify interventions to improve survival in this population.

Copyright $\odot 2009$ S. Karger AG, Basel

\section{Introduction}

Pneumonia is a common illness associated with substantial morbidity and mortality, and is a leading cause of death in the United States [1]. It is responsible for approximately 1.4 million hospitalizations and, in combi-

Dedicated in memory of Dr. Richard K. Root.
Dr. Laura Cecere

Division of Pulmonary and Critical Care, Department of Medicine

University of Washington School of Medicine, 1100 Olive Way Suite 1400

Campus Box 358280, Seattle, WA 98101 (USA)

Tel. +1 206207 4191, Fax +1 206768 5343, E-Mail lcecere@ u.washington.edu 
nation with influenza, results in almost 60,000 deaths annually $[1,2]$. Community-acquired pneumonia (CAP) is defined as pneumonia which develops in patients outside the hospital setting. Healthcare-associated pneumonia (HCAP) is a more recently described entity which includes patients admitted from the community with pneumonia who have an increased risk of infection from the same Gram-negative and drug-resistant organisms usually associated with nosocomial infections [3-6]. Patients hospitalized with HCAP have been shown to have increased hospital mortality compared to patients admitted with CAP [4-6].

Previous studies showing increased mortality in the years following recovery from an episode of pneumonia have concentrated on patients with a diagnosis of CAP and have included mostly older patients [7-13]. To our knowledge, no study to date has examined long-term outcomes in patients hospitalized with HCAP. Only three studies have examined survival $>4$ years after a diagnosis of CAP $[7,10,13]$, with only one study including patients under age 60 at the time of diagnosis [7]. Thus, little is known regarding the long-term significance of an episode of pneumonia in younger adults.

Patients admitted to public hospitals with pneumonia tend to be young and have high rates of homelessness, alcohol use, and intravenous drug abuse [14-16]. We have shown that these and other factors may influence the decision to admit patients with 'low-risk' pneumonia (as defined by pneumonia severity index, PSI, classes I-III [17]) who might otherwise be considered candidates for treatment in outpatient settings [15]. Very little is known about the impact of these socioeconomic factors on survival after discharge, however, and to date no study has focused solely on outcomes of younger patients with prevalent socioeconomic disadvantage.

The goal of this study was to describe long-term survival after discharge in a well-characterized cohort of patients admitted to a public teaching hospital with a diagnosis of pneumonia. We sought to compare survival in patients admitted with CAP to that of patients who met the criteria for HCAP and to identify clinical and sociodemographic factors associated with outcomes in each group.

\section{Patients and Methods}

\section{Patients and Procedures}

This is a cohort study of patients admitted between June 1, 1994, and May 30, 1996, to Harborview Medical Center, an urban public teaching hospital affiliated with the University of Wash- ington in Seattle. This cohort has been described in detail previously [14]. All admissions during this time period were screened for study inclusion by determining whether or not they met the criteria for CAP as it was defined at the time of the study $[17,18]$. Patients were excluded if they were $<18$ years old, hospitalized within the past week, transferred from another hospital, admitted for terminal care, or unable to provide consent. In the present analysis, patients were reclassified as having HCAP if they were known to have been treated for pneumonia or hospitalized in the 90 days prior to admission, resided in a skilled-nursing facility or other institution, received chronic dialysis, or were known to be immunocompromised (due to HIV infection, neutropenia in the past 2 weeks, use of $\geq 20 \mathrm{mg}$ of prednisone per day, or other immunosuppressant drugs) at the time of admission [3]. Only patients surviving their initial hospitalization for pneumonia were included in the present analysis, with repeat admissions excluded from this study.

The follow-up period began at the time of discharge and was continued until death or December 31, 2002. Informed consent was obtained from all patients subjected to the initial study procedures. A waiver of consent was obtained from the University of Washington Human Subjects Committee to link patient records to death certificates. The University of Washington Human Subjects Committee approved all study protocols and analyses.

\section{Identification of Clinical and Demographic Data}

Clinical information and sociodemographic data were obtained by patient interview and review of the medical record. All study data were collected on a standardized data form, then double entered into a database. Discrepancies were reconciled by repeat examination of the primary record. The PSI was calculated at the time of admission for each subject and categorized as 'low risk' for PSI classes I-III and 'high risk' for PSI classes IV-V [17].

\section{Identification of Deaths}

Deaths during the follow-up period were determined utilizing data from the Washington State Death Certificate Data Files compiled by the Center for Health Statistics of the Washington State Department of Health [19]. We obtained death registry information from June 1, 1994, to December 31, 2002. First and last names (as well as middle initials and address when available) and birthdates were used to link study subjects with data from the death registry. In cases in which there was not a match for both names and birthdates, we assumed that the patient was alive at the end of the follow-up period. To test the validity of this method, all deaths that occurred during the index hospitalization were confirmed as matching with the death certificate data files.

Cause of death was determined from death certificates. Individual diagnoses were grouped into broad categories of chronic lung disease, human immunodeficiency virus (HIV) related, malignancy, cardiovascular disease, trauma, and other.

\section{Statistical Analysis}

Continuous variables were compared between groups using Student's t tests or Mann-Whitney rank sum tests, as appropriate. $\chi^{2}$ tests were used to compare nominal data. Survival was assessed using the Kaplan-Meier product limit estimator and the log rank statistic. Pre-specified subgroups included those patients $\geq 65$ years of age and those who would now be classified as having 


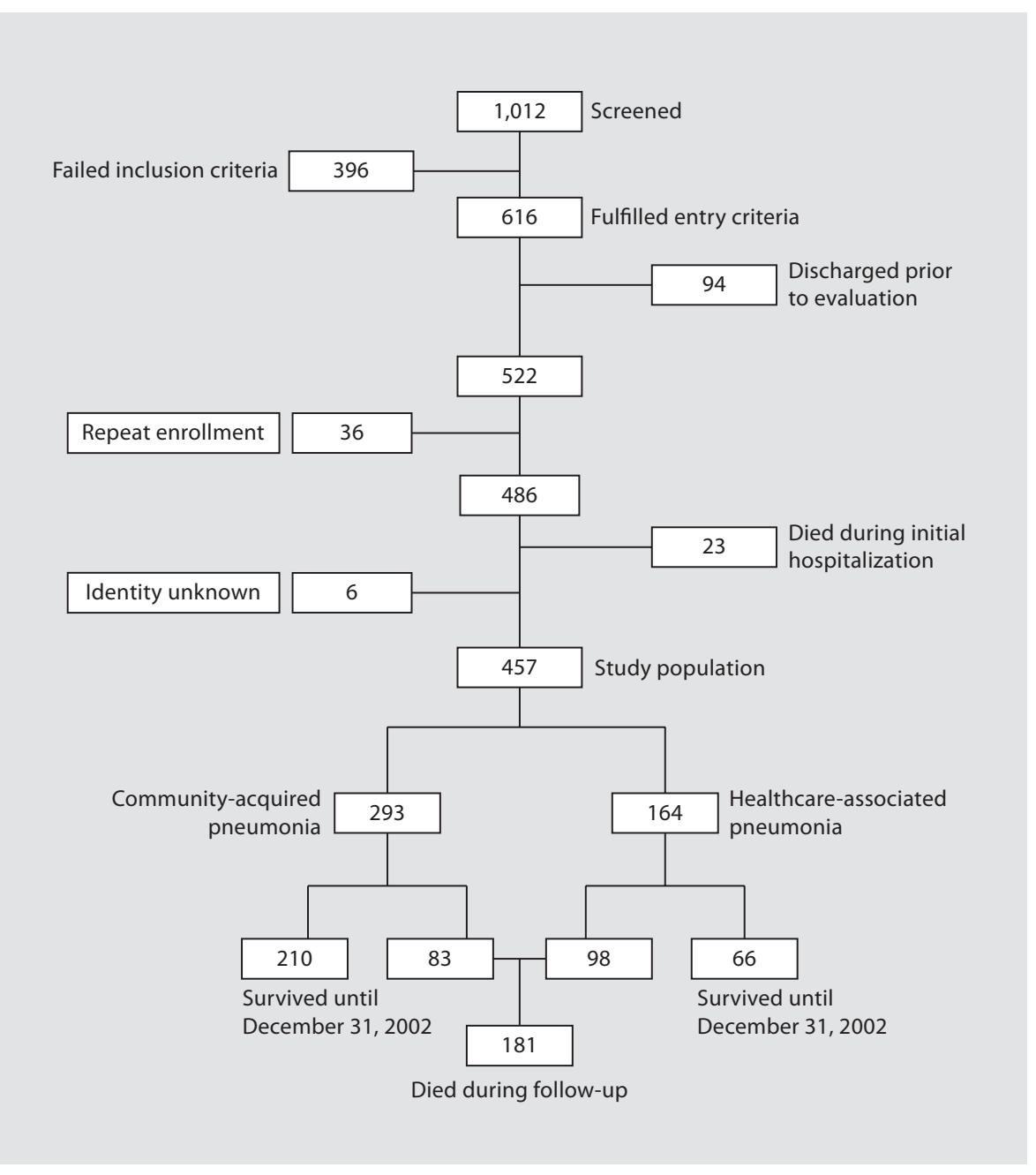

Fig. 1. Study population.

HCAP based on current criteria [3]. The Cox proportional hazard model was used to estimate differences in survival after controlling for confounders [20]. The proportional hazards assumption was tested using graphical inspection of observed and expected survival curves and log-log negative plots [21]. Confounders were identified by evaluating the effect of clinically relevant variables on the effect of age on survival and forced in manually in a forward approach [22]. Wald $\chi^{2}$ tests were used to evaluate the effect of individual variables on survival. Clinically relevant interactions were decided a priori. Using a similar methodology to that employed by Mortensen et al. [7], United States life table data were used to calculate expected 5-year survival based on age and gender and comparison was made to that observed in our study population [23]. The standardized mortality ratio, defined as the comparison of observed deaths in our population to expected deaths based on United States death rates, was then computed [23, 24]. A two-tailed $p$ value $<0.05$ was considered significant. We used SAS 9.1 (SAS Institute, Cary, N.C., USA) for Windows and STATA 8.0 software (STATA, Chicago, Ill., USA).

\section{Results}

Of the 1,012 hospitalizations screened, 522 fulfilled entry criteria and were included in the original study (fig. 1). Thirty-six of these admissions represented repeat hospitalizations, leaving 486 individual subjects eligible for this analysis. Six patients with no known name were excluded from this study. Of the remaining 480 individual patients, 457 (95\% confidence interval, CI, 93-97\%) survived to hospital discharge and made up the cohort assessed for long-term outcome. Of these 457 patients, 164 (36\%, 95\% CI 32-40\%) were re-categorized as having HCAP for the current analysis, with the remaining 293 (64\%, 95\% CI 60-69\%) classified as cases of CAP. Twentyone patients were known to have been hospitalized sometime in the past year, but with unknown dates of admission. These patients were classified as having CAP for the 
purpose of this study. Additional analyses performed with omission of these patients from the cohort showed no differences in outcome.

\section{Clinical and Sociodemographic Characteristics}

This cohort was predominantly young, with 378 (83\%, 95\% CI 79-86\%) patients under age 65 at the time of admission and a mean age of 47 years (SD 16 years; table 1 ). The majority of patients were male $(\mathrm{n}=354,77 \%)$. One hundred and twenty-four (27\%) patients were homeless. One hundred and eighty-nine (41\%) had a prior diagnosis of alcoholism or a positive blood alcohol level at the time of admission, and 75 (16\%) reported using injection drugs. With regard to severity of illness, the majority of patients were classified as having 'low-risk' pneumonia, with 292 (64\%) falling into PSI classes I-III and 165 (36\%) defined as having 'high-risk' pneumonia (PSI classes IV-V) [17].

Patients with CAP were significantly more likely to be homeless (35 vs. 13\%), use tobacco (70 vs. $58 \%$ ), abuse alcohol or intravenous drugs (53 vs. $39 \%$ ), to have a diagnosis of obstructive lung disease ( 27 vs. $18 \%$ ), and to be designated as having self-pay insurance status (65 vs. $55 \%$ ) than those with HCAP. Patients categorized as having HCAP were significantly more likely to be Caucasian (60 vs. $47 \%$ ), have cerebrovascular disease (10 vs. $4 \%$ ), have 'high-risk' pneumonia ( 45 vs. $31 \%$ ), and to be insured by Medicare ( 34 vs. $21 \%$ ). In patients under the age of 65 years, these same differences were seen. In patients over the age of 65 years, however, the two groups were more similar. In this age group, patients admitted with HCAP were only significantly more likely to be insured by Medicaid ( 94 vs. $77 \%$ ).

\section{Survival of the Cohort}

Of the 457 patients surviving their index hospitalization, 181 (40\%, 95\% CI 35-44\%) died by the end of our follow-up period. Mean follow-up time of survivors was 7.6 years (range 6.6-8.6 years); for non-survivors, the median time to death was 1.8 years (range $0.0-8.2$ years), with 1 patient dying within $24 \mathrm{~h}$ of discharge. Figure $2 \mathrm{a}$ shows a Kaplan-Meier plot of survival for the study cohort. Overall survival in this group is poor, with a 2 -year survival rate of $78 \%$ (95\% CI $74-82 \%)$, and a 5 -year survival rate of $67 \%$ (95\% CI 62-70\%). When analyzed based on age (dichotomized to $<65$ vs. $\geq 65$ years) and pneumonia type, older patients and those with HCAP were noted to have an expected increased mortality (fig. 2b, c). However, the mortality rate seen in young patients admitted with both CAP and HCAP was higher than would be expected. Of the 181 deaths that occurred in the entire co- hort, 120 of these deaths occurred in patients $<65$ years. Fifty of these deaths occurred in young patients admitted with CAP, corresponding to a death rate of $20 \%$ in this group by the end of the follow-up period. Of the $132 \mathrm{pa}-$ tients $<65$ years admitted with HCAP, 70 deaths occurred in the follow-up period (53\% death rate), with 19 of these deaths $(27 \%)$ occurring in patients without known HIV infection. In patients $<65$ years whose only risk factor for HCAP was treatment for pneumonia or hospitalization in the last 90 days, 4 of 13 patients died during the follow-up period (death rate of $31 \%$ ). As shown in figure $3 a$, overall 2 -year survival seen in patients $<65$ years admitted with HCAP was less than that seen in patients aged $\geq 65$ years with CAP (61 vs. $68 \%$ ). Five-year survival between the two groups remained similar (51\% in young patients in HCAP and $47 \%$ in older patients with CAP). As a whole, there was no significant difference in estimated long-term survival of patients with known HIV infection compared to HIV-negative patients admitted with HCAP (fig. 3b).

\section{Cause of Death}

HIV and cardiovascular disease combined were responsible for $50 \%$ of deaths in our cohort. Chronic lung disease was responsible for only $12 \%$ of the total deaths observed. Despite the high rates of alcohol and drug abuse observed in our population, trauma-related injury resulted in only $4 \%$ of the deaths in this cohort. In patients admitted with CAP, cardiovascular disease was the leading cause of death (35\%). Complications of HIV infection were responsible for $46 \%$ of the deaths in those admitted with HCAP.

\section{Independent Factors Associated with Outcomes}

Table 2 shows clinical and demographic factors significantly associated with death in the univariate analysis. Homelessness and different categories of substance abuse were associated with a reduced hazard of death; however, this association was confounded by age. These variables were not statistically significant in the multivariate model. Factors independently associated with death in the multivariate model are summarized in table 3. Age $>65$ years, a diagnosis of HCAP, the presence of HIV infection before admission, use of immunosuppressant drugs other than prednisone, 'high-risk' pneumonia upon presentation, and cardiovascular disease were all predictors of increased mortality after discharge in our patient population. In the subgroup of patients admitted with CAP, age $>65$ years, 'high-risk' pneumonia and cardiovascular disease were all associated with increased mortality during follow-up. In patients now cat- 
Table 1. Clinical and sociodemographic characteristics of survivors of hospitalization for pneumonia

\begin{tabular}{|c|c|c|c|c|}
\hline Characteristics & Study cohort $(\mathrm{n}=457)$ & CAP $(n=293)$ & $\operatorname{HCAP}(n=164)$ & $\mathrm{p}$ value \\
\hline Mean age, years & $47.3 \pm 16.4$ & $47.9 \pm 15.8$ & $46.1 \pm 18.4$ & 0.180 \\
\hline$\geq 65$ years & $79(17)$ & $47(16)$ & $32(20)$ & 0.347 \\
\hline By decade & & & & 0.004 \\
\hline$<30$ years & $55(12)$ & $36(12)$ & $19(12)$ & \\
\hline $30-39$ years & $125(27)$ & $66(23)$ & $59(36)$ & \\
\hline $40-49$ years & $109(24)$ & $71(24)$ & $38(23)$ & \\
\hline $50-59$ years & $65(14)$ & $54(18)$ & $11(7)$ & \\
\hline $60-69$ years & $43(9)$ & $31(11)$ & $12(7)$ & \\
\hline $70-79$ years & $44(10)$ & $26(9)$ & $18(11)$ & \\
\hline$\geq 80$ years & $16(4)$ & $9(3)$ & $7(4)$ & \\
\hline \multicolumn{5}{|l|}{ Gender } \\
\hline Male & $354(77)$ & $220(75)$ & $134(82)$ & 0.104 \\
\hline \multicolumn{5}{|l|}{ Race } \\
\hline Caucasian & $238(52)$ & $139(47)$ & $99(60)$ & 0.008 \\
\hline \multicolumn{5}{|l|}{ Residence } \\
\hline Homeless & $124(27)$ & $102(35)$ & $22(13)$ & 0.001 \\
\hline SNF or other institution & $49(11)$ & NA & $49(30)$ & $<0.001$ \\
\hline \multicolumn{5}{|l|}{ Insurance status } \\
\hline Medicare & $117(26)$ & $62(21)$ & $55(34)$ & 0.004 \\
\hline Medicaid & $396(87)$ & $251(86)$ & $145(88)$ & 0.407 \\
\hline Self-pay & $281(61)$ & $190(65)$ & $91(55)$ & 0.049 \\
\hline Community insurance & $27(6)$ & $19(6)$ & $8(5)$ & 0.485 \\
\hline $\mathrm{HMO} / \mathrm{PPO}$ & $4(1)$ & $3(1)$ & $1(1)$ & 0.648 \\
\hline \multicolumn{5}{|l|}{ Alcohol use } \\
\hline History of alcoholism and/or BAC & $189(41)$ & $141(48)$ & $48(29)$ & $<0.001$ \\
\hline \multicolumn{5}{|l|}{ Substance abuse } \\
\hline Intravenous drug use & $75(16)$ & $51(17)$ & $24(15)$ & 0.443 \\
\hline Marijuana & $41(9)$ & $27(9)$ & $14(9)$ & 0.808 \\
\hline Crack & $72(16)$ & $51(17)$ & $21(13)$ & 0.195 \\
\hline Combined substance abuse & $121(26)$ & $81(28)$ & $40(24)$ & 0.449 \\
\hline Alcohol or intravenous drug use & $219(48)$ & $155(53)$ & $64(39)$ & 0.004 \\
\hline Ongoing tobacco use (within the last 30 days) & $299(65)$ & $204(70)$ & $95(58)$ & 0.012 \\
\hline \multicolumn{5}{|l|}{ Comorbid illness } \\
\hline Immunocompromised & $95(21)$ & 0 & $95(58)$ & $<0.001$ \\
\hline HIV infection & $89(19)$ & 0 & $89(54)$ & $<0.001$ \\
\hline Neutropenia in past 2 weeks & $2(1)$ & 0 & $2(1)$ & 0.058 \\
\hline Prednisone ( $\geq 20 \mathrm{mg}$ daily) & $7(2)$ & 0 & $7(4)$ & $<0.001$ \\
\hline Other immunosuppressant & $5(1)$ & 0 & $5(3)$ & 0.003 \\
\hline Malignancy & $37(8)$ & $19(6)$ & $18(11)$ & 0.091 \\
\hline Cirrhosis & $20(4)$ & $12(4)$ & $8(5)$ & 0.695 \\
\hline Dialysis & $8(2)$ & 0 & $8(5)$ & $<0.001$ \\
\hline Cardiovascular disease & $108(24)$ & $66(23)$ & $42(26)$ & 0.457 \\
\hline Cerebrovascular disease & $29(6)$ & $13(4)$ & $16(10)$ & 0.025 \\
\hline Diabetes & $27(6)$ & $21(7)$ & $6(4)$ & 0.127 \\
\hline Obstructive lung disease & $108(24)$ & $78(27)$ & $30(18)$ & 0.044 \\
\hline Hospitalized or treated for pneumonia in previous 90 days & $39(9)$ & 0 & $39(24)$ & $<0.001$ \\
\hline \multicolumn{5}{|l|}{ PSI class } \\
\hline Low risk (I-III) & $292(64)$ & $201(92)$ & $91(55)$ & 0.005 \\
\hline High risk $(\mathrm{IV}, \mathrm{V})$ & $165(36)$ & $92(31)$ & $73(45)$ & 0.005 \\
\hline
\end{tabular}

Data are presented as means \pm SD and numbers (\%). SNF = Skilled-nursing facility; HMO/PPO = health maintenance organization/preferred provider organization; $\mathrm{BAC}=$ blood alcohol concentration (any alcohol detected). 


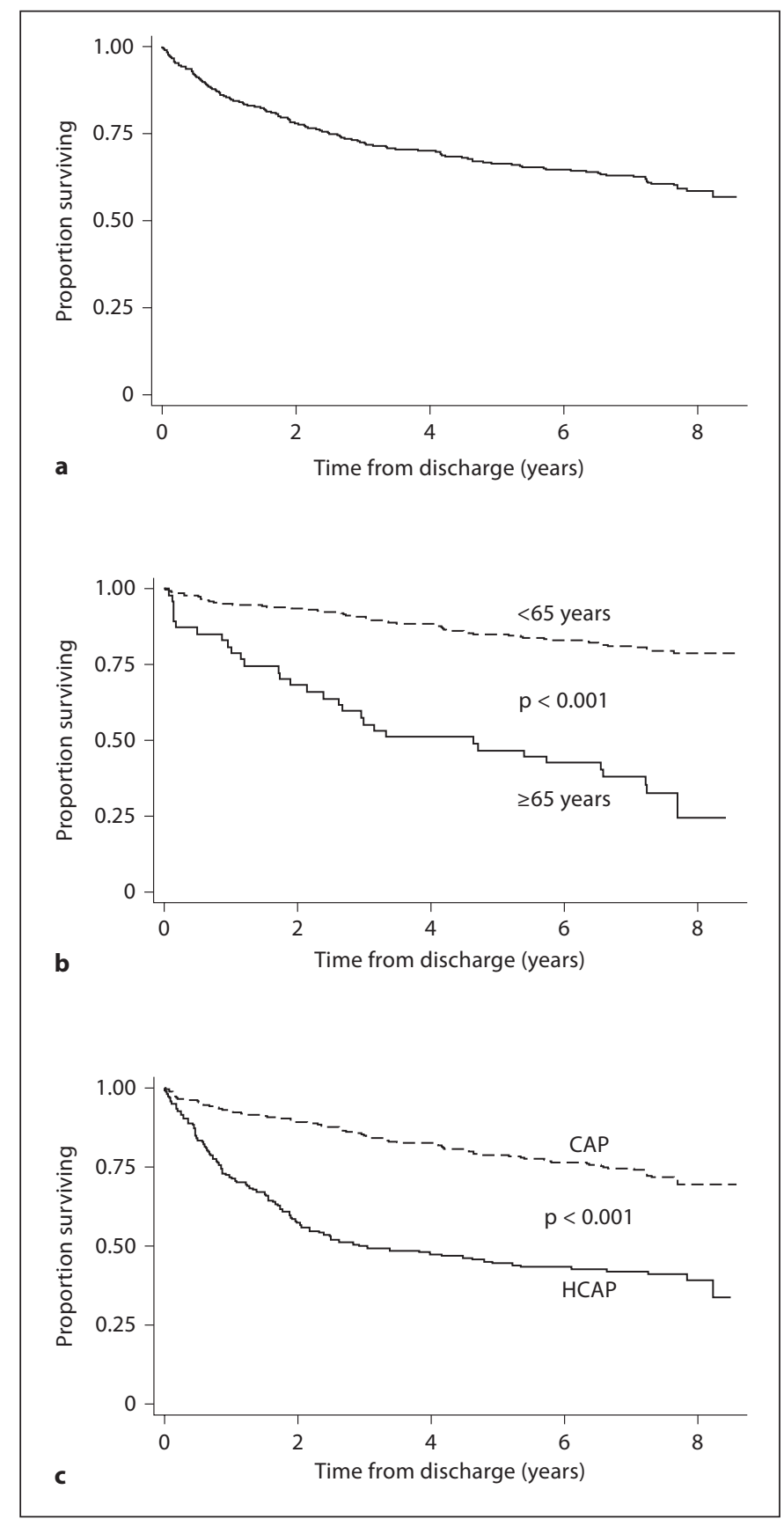

Fig. 2. Kaplan-Meier survival estimates (a-c) for patients surviving hospitalization for pneumonia by age (b) and pneumonia type (c).

egorized as having HCAP, the predictors of increased mortality included increasing age, use of immunosuppressant drugs, neutropenia, and cerebrovascular disease. Subgroup analysis of patients under age 65 yielded similar results in each category of pneumonia with the exceptions that diabetes was associated with increased

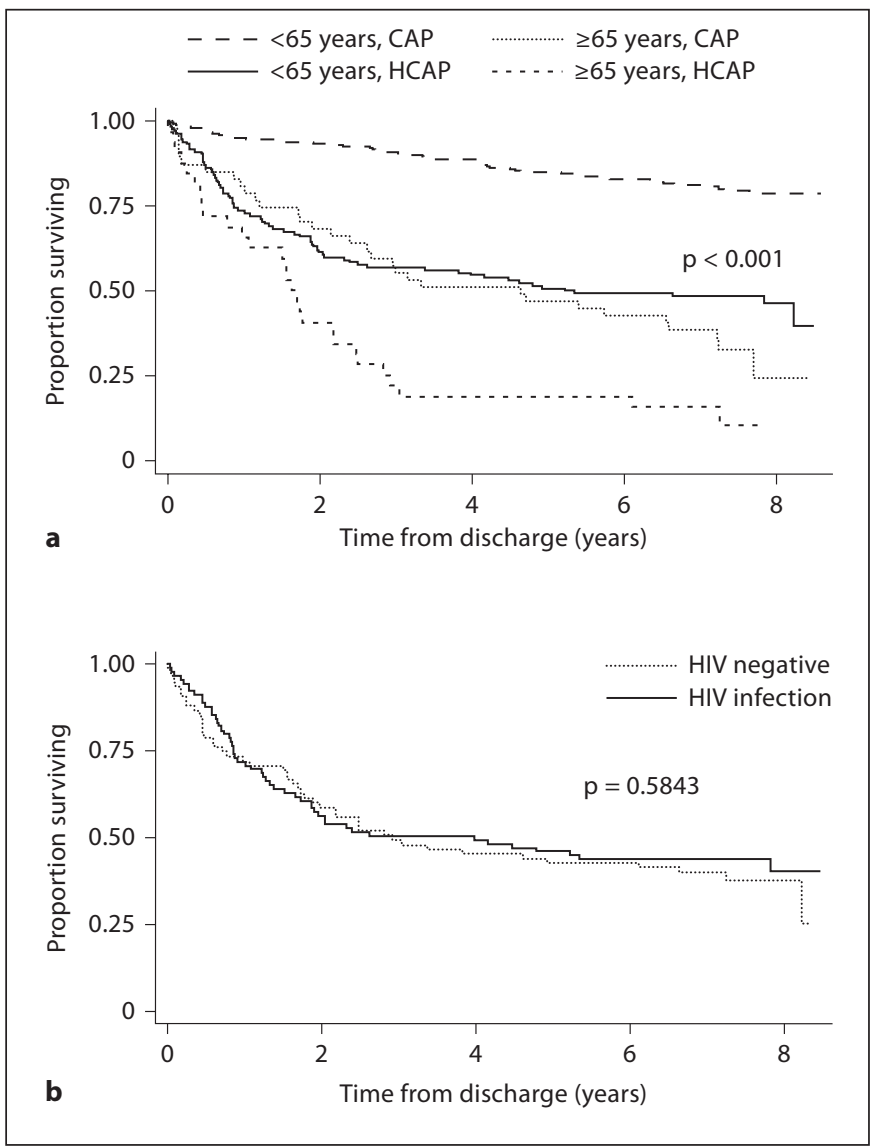

Fig. 3. Kaplan-Meier survival estimates for patients surviving hospitalization for pneumonia by age and pneumonia type (a) and by admission HIV status (b).

mortality after discharge in young patients admitted with CAP (hazard ratio 2.22 , 95\% CI 1.03-4.77) and self-pay status was associated with decreased mortality in patients with HCAP (hazard ratio 0.51, 95\% CI 0.31-0.84).

\section{Comparison with National Data}

When compared with data derived from US life table data, our cohort shows a higher than expected mortality by categorical age (table 4, fig. 4). Although this difference is most significant in patients admitted with HCAP, decreased survival is seen even in CAP-admitted patients in almost every age group. The standardized mortality ratio for those patients admitted with CAP was 3.9, while that of patients admitted with HCAP was 9.9. Overall, an admission for pneumonia was associated with a standard mortality ratio of 6.0 , representing a 6 -fold increase over what would be expected in a group of patients of the same age and gender.

Respiration 2010;79:128-136 
Table 2. Univariate model: characteristics associated with longterm mortality in patients admitted with pneumonia

\begin{tabular}{|c|c|c|c|}
\hline Characteristics & HR & $95 \% \mathrm{CI}$ & $\mathrm{p}$ value \\
\hline \multicolumn{4}{|l|}{ Age } \\
\hline$\geq 65$ years & 3.52 & $2.58-4.80$ & $<0.001$ \\
\hline \multicolumn{4}{|l|}{ By decade: } \\
\hline$<30$ years & Ref. & & \\
\hline $30-39$ years & 1.11 & $0.58-2.11$ & 0.748 \\
\hline $40-49$ years & 1.52 & $0.81-2.85$ & 0.194 \\
\hline $50-59$ years & 1.44 & $0.73-2.85$ & 0.292 \\
\hline $60-69$ years & 2.98 & $1.52-5.85$ & 0.002 \\
\hline $70-79$ years & 4.85 & $2.56-9.18$ & $<0.001$ \\
\hline$\geq 80$ years & 8.11 & $3.85-17.1$ & $<0.001$ \\
\hline \multicolumn{4}{|l|}{ Gender } \\
\hline Male & 1.22 & $0.85-1.76$ & 0.281 \\
\hline \multicolumn{4}{|l|}{ Race } \\
\hline Caucasian & 1.41 & $1.05-1.89$ & 0.023 \\
\hline \multicolumn{4}{|l|}{ Residence } \\
\hline Homeless & 0.48 & $0.32-0.70$ & $<0.001$ \\
\hline SNF or other institution & 2.46 & $1.68-3.58$ & $<0.001$ \\
\hline \multicolumn{4}{|l|}{ Insurance status } \\
\hline Medicare & 2.48 & $1.84-3.35$ & $<0.001$ \\
\hline Medicaid & 1.22 & $0.78-1.90$ & 0.380 \\
\hline Self-pay & 0.56 & $0.42-0.75$ & $<0.001$ \\
\hline Community insurance & 0.78 & $0.40-1.52$ & 0.461 \\
\hline $\mathrm{HMO} / \mathrm{PPO}$ & 1.84 & $0.59-5.77$ & 0.294 \\
\hline \multicolumn{4}{|l|}{ Alcohol use } \\
\hline History of alcoholism and/or BAC & 0.73 & $0.54-0.98$ & 0.038 \\
\hline \multicolumn{4}{|l|}{ Substance abuse } \\
\hline Intravenous drug use & 0.65 & $0.41-1.01$ & 0.056 \\
\hline Marijuana & 0.83 & $0.48-1.43$ & 0.499 \\
\hline Crack & 0.48 & $0.29-0.80$ & 0.004 \\
\hline Combined substance abuse & 0.57 & $0.39-0.83$ & 0.003 \\
\hline Alcohol or intravenous drug use & 0.72 & $0.54-0.97$ & 0.030 \\
\hline \multicolumn{4}{|l|}{ Ongoing tobacco use } \\
\hline (within the last 30 days) & 0.95 & $0.70-1.30$ & 0.768 \\
\hline \multicolumn{4}{|l|}{ Comorbid illness } \\
\hline Immunocompromised & 2.13 & $1.55-2.93$ & $<0.001$ \\
\hline HIV infection & 2.11 & $1.52-2.92$ & $<0.001$ \\
\hline Neutropenia in past 2 weeks & 28.20 & $6.68-119.00$ & $<0.001$ \\
\hline Prednisone ( $\geq 20 \mathrm{mg}$ daily) & 1.39 & $0.44-4.36$ & 0.571 \\
\hline Other immunosuppressant & 9.77 & $3.91-24.40$ & $<0.001$ \\
\hline Malignancy & 2.70 & $1.78-4.09$ & $<0.001$ \\
\hline Cirrhosis & 1.31 & $0.69-2.48$ & 0.405 \\
\hline Dialysis & 4.18 & $1.95-8.93$ & $<0.001$ \\
\hline Cardiovascular disease & 2.47 & $1.83-3.34$ & $<0.001$ \\
\hline Cerebrovascular disease & 2.27 & $1.41-3.65$ & 0.001 \\
\hline Diabetes & 1.71 & $1.03-2.86$ & 0.040 \\
\hline Obstructive lung disease & 1.18 & $0.85-1.64$ & 0.331 \\
\hline \multicolumn{4}{|l|}{ Hospitalized or treated for pneumonia } \\
\hline in previous 90 days & 1.94 & $1.24-3.03$ & 0.004 \\
\hline \multicolumn{4}{|l|}{ PSI class } \\
\hline High risk (IV, V) & 3.34 & $2.48-4.49$ & $<0.001$ \\
\hline HCAP & 2.97 & $2.21-3.99$ & $<0.001$ \\
\hline
\end{tabular}

Unless otherwise indicated, reference value assumed to be the absence of the condition.

$\mathrm{SNF}=$ Skilled-nursing facility; $\mathrm{HMO} / \mathrm{PPO}=$ health maintenance organization/preferred provider organization; $\mathrm{BAC}=$ blood alcohol concentration (any alcohol detected); HR = hazard ratio.
Table 3. Multivariate analysis: characteristics independently associated with long-term mortality

\begin{tabular}{llll}
\hline Comorbidity & HR & $95 \%$ CI & p value \\
\hline Aged $\geq 65$ years & 2.30 & $1.54-3.43$ & $<0.001$ \\
HCAP & 1.97 & $1.36-2.87$ & $<0.001$ \\
High-risk pneumonia (PSI IV-V) & 2.13 & $1.51-3.02$ & $<0.001$ \\
Immunosuppressant drug $^{\mathrm{a}}$ & 8.04 & $3.11-20.76$ & $<0.001$ \\
HIV infection & 1.67 & $1.08-2.59$ & 0.021 \\
Cardiovascular disease & 1.50 & $1.05-2.14$ & 0.024 \\
\hline
\end{tabular}

Unless otherwise indicated, reference value assumed to be the absence of this condition. $\mathrm{HR}=$ Hazard ratio.

${ }^{\text {a }}$ Other than prednisone.

\section{Discussion}

The results of this study show that hospitalization for pneumonia at our urban county hospital is associated with poor long-term survival. Forty percent of patients died during the follow-up period, with $66 \%$ of the deaths occurring in patients under the age of 65 at the time of admission. Previous studies have demonstrated that surviving an episode of pneumonia is associated with a subsequent increased mortality [7-13]. However, most of these studies have concentrated on outcomes of older patients and have not distinguished between cases of CAP and HCAP. Our study is unique in that the majority of the patients in our cohort (73\%) were under age 65 at the time of admission. It is also the first that we know of to compare long-term outcomes of patients admitted with HCAP with those of patients admitted with CAP.

We found a diagnosis of HCAP to be an independent predictor of increased long-term mortality in this patient population. Patients over the age of 65 with HCAP had only a $24 \%$ survival rate during the 5 years following discharge. In addition, more than half of the 132 patients under the age of 65 admitted with HCAP died during the follow-up. A previous study has shown patients admitted to an urban teaching hospital with HCAP are significantly more likely to die during their initial hospitalization than patients with CAP [6]. In a separate study, Kolleff et al. [5] found the in-hospital mortality rate in patients admitted with HCAP to be significantly higher than that for CAP, and comparable to that of hospital-acquired pneumonia. The results of our study show the increased risk of death may continue for several years after discharge in the patients surviving their initial hospitalization. While some of this increase in mortality might be expected due 
Table 4. Five-year survival in survivors of hospitalization for pneumonia

\begin{tabular}{|c|c|c|c|c|c|c|c|c|c|}
\hline \multirow[t]{2}{*}{ Age } & \multicolumn{3}{|c|}{ Combined cohort } & \multicolumn{3}{|l|}{ CAP } & \multicolumn{3}{|l|}{ HCAP } \\
\hline & survival, \% & $\begin{array}{l}\text { expected } \\
\text { survival, \% }\end{array}$ & SMR & survival, $\%$ & $\begin{array}{l}\text { expected } \\
\text { survival, \% }\end{array}$ & SMR & survival, $\%$ & $\begin{array}{l}\text { expected } \\
\text { survival, \% }\end{array}$ & SMR \\
\hline $18-29$ years & $42(76.4)$ & $55(99.5)$ & 43.4 & $32(88.9)$ & $36(99.5)$ & 20.0 & $10(52.6)$ & $19(99.5)$ & 90.0 \\
\hline $30-39$ years & $93(74.4)$ & $124(99.0)$ & 26.7 & $62(93.9)$ & $65(99.0)$ & 5.7 & $31(52.5)$ & $58(99.0)$ & 46.7 \\
\hline $40-49$ years & $79(72.5)$ & $107(98.3)$ & 15.8 & $61(85.9)$ & $70(98.3)$ & 8.3 & $18(47.4)$ & $37(98.3)$ & 33.3 \\
\hline $50-59$ years & $49(75.4)$ & $63(96.3)$ & 6.7 & $42(77.8)$ & $52(96.3)$ & 6.0 & $7(63.6)$ & $11(96.3)$ & 10.0 \\
\hline $60-69$ years & $23(53.5)$ & $39(90.9)$ & 5.1 & $21(67.7)$ & $28(90.9)$ & 3.6 & $2(16.7)$ & $10(90.9)$ & 4.8 \\
\hline $70-79$ years & $13(29.6)$ & $36(81.2)$ & 3.7 & $8(30.8)$ & $21(81.2)$ & 3.7 & $5(27.8)$ & $15(81.2)$ & 3.8 \\
\hline $80-104$ years & $5(31.3)$ & $8.5(53.3)$ & 1.5 & $5(55.6)$ & $4.8(53.3)$ & 0.9 & $0(0)$ & $4(53.3)$ & 2.1 \\
\hline Overall & $304(66.5)$ & $432(94.4)$ & 6.0 & $231(78.8)$ & $277(94.4)$ & 3.9 & $73(44.5)$ & $155(94.4)$ & 9.9 \\
\hline
\end{tabular}

Comparison was made to expected survival based on US life tables for subjects of similar age and gender. SMR = Standardized mortality ratio.

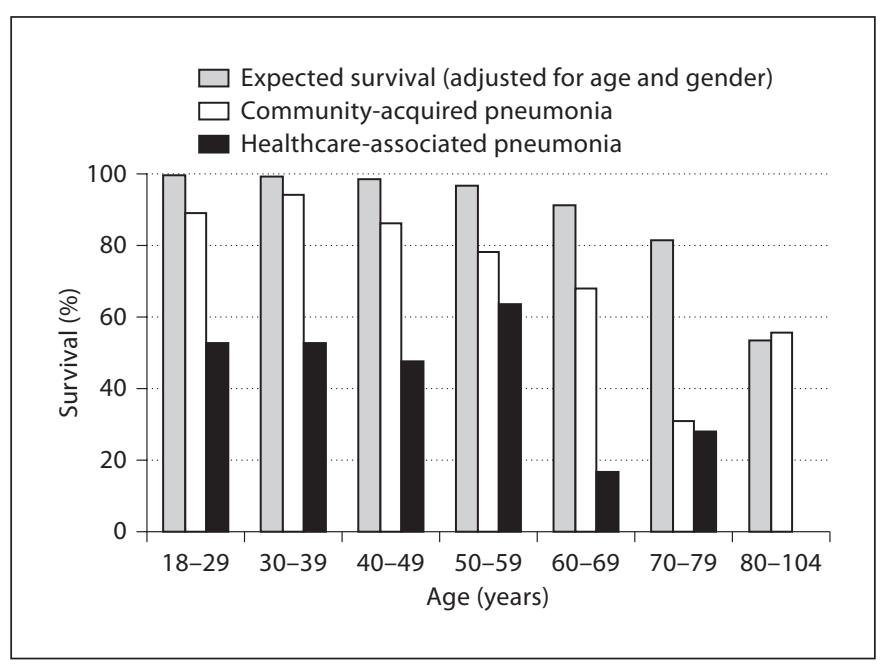

Fig. 4. Observed versus expected 5-year survival (based on US life table data, adjusted for age and gender) after hospitalization for pneumonia.

to ongoing risk factors (dialysis, skilled-nursing home residence, or immunosuppression), it is interesting to note that almost a third of the patients under age 65 whose only risk factor for HCAP was recent hospitalization or treatment for pneumonia died during the follow-up period.

An important question to be answered is whether unique socioeconomic characteristics of patients admitted to urban county hospitals contribute to the mortality rate in these young patients. Although a majority of patients in this study had 'low-risk' pneumonia, as defined by the PSI [17], many had social factors that precluded treatment in an ambulatory setting [15]. Despite this, abuse of alcohol, intravenous drug use, and homelessness were not independently associated with an increased risk of death. Thus, the presence of these risk factors alone does not explain the overall increase in mortality seen in this patient population.

It may be that hospitalization for pneumonia, particularly HCAP and to a lesser degree CAP, may itself be a marker for poor long-term survival. This is supported by data from two previous studies which have shown an independent correlation between a diagnosis of CAP in elderly patients and increased mortality $[9,13]$.

Several limitations to this study should be acknowledged. First, because HCAP was not formally recognized at the time of initial study enrollment, patients were not identified upon admission as having HCAP as they would be today. Because of this, we do not have complete information on patient antibiotic use in the 90 days prior to admission. However, those with the most serious infectious illnesses were likely to have been hospitalized and would therefore be appropriately classified as having HCAP for this study. Because we had information regarding immunosuppressed patients and those treated for prior pneumonia during this same time, we would expect the resulting overall misclassification due to antibiotic use alone is likely small. Second, as the initial study design did not include a control group, we compared overall survival in our cohort to a population derived from national life table data. As a result, we were unable to determine the true impact of the underlying socioeconomic factors of an urban public hospital population on patient outcome. Third, we were unable to determine the vital status of all patients enrolled. Instead, we ascertained death only in patients known to have died in Washington State at the 
end of the follow-up period. This may lead to an underestimation of death due to the high number of homeless patients in our cohort, as individuals without a permanent residence may be more likely to move outside the state. Thus, the bias of our study would be to underestimate the true death rate, making our findings even more significant. Lastly, because the study was performed at a single institution, the results may not be applicable to patient populations other than those admitted to a public teaching hospital. A previous study looking at adjusted mortality rates based on hospital ownership and teaching status showed an increased risk of death in the 6 months after discharge for patients admitted for all causes to public teaching hospitals, when compared to several other hospital types [25]. Once adjusted for patient risk, however, this difference in mortality accounted for only 9 more patients per 1,000 when compared to hospitals having the best outcomes, indicating hospital type likely does not have a significant impact on long-term outcomes.
In summary, admission for pneumonia is associated with increased mortality even in relatively young patients admitted to an urban county hospital. This is particularly true of patients admitted with HCAP, even those who are under age 65 and are HIV negative at the time of admission. The results of our study emphasize the importance of identifying these patients early and understanding the potential for markedly decreased survival not only in the short-term, but also in the years following discharge. Future studies are warranted to find areas of intervention to help improve survival after discharge.

\section{Acknowledgment}

This study was supported by NIH/NHLBI Grant 1 K23 HL72017-04.

\section{References}

$\checkmark 1$ Heron M: Deaths: leading causes for 2004. Natl Vital Stat Rep 2007;56:1-96.

2 DeFrances CJ, Hall MJ: 2005 National Hospital Discharge Survey. Adv Data2007;385: $1-20$.

-3 Kollef MH, Morrow LE, Baughman RP, et al: Health care-associated pneumonia (HCAP): a critical appraisal to improve identification, management, and outcomes - proceedings of the HCAP summit. Clin Infect Dis 2008; 46:S296-S334.

4 Carratala J, Mykietiuk A, Fernandez-Sabe N, et al: Heath care-associated pneumonia requiring hospital admission: epidemiology, antibiotic therapy and clinical outcomes. Arch Intern Med 2007;167:1393-1399.

5 Kolleff MH, Shorr A, Tabak YP, et al: Epidemiology and outcomes of health-care-associated pneumonia: results from a large US database of culture-positive pneumonia. Chest 2005;128:3854-3862.

6 Micek ST, Kollef KE, Reichley RM, et al: Health care-associated pneumonia and community-acquired pneumonia: a single-center experience. Antimicrob Agents Chemother 2007;51:3568-3573.

-7 Mortensen EM, Kapoor WN, Chang CC, et al: Assessment of mortality after long-term follow-up of patients with community-acquired pneumonia. Clin Infect Dis 2003;37: 1617-1624.

-8 Hedlund JU, Ortqvist AB, Kalin ME, et al: Factors of importance for the long term prognosis after hospital treated pneumonia. Thorax 1993;48:785-789.
9 Kaplan V, Clermont G, Griffin MF, et al: Pneumonia: still the old man's friend? Arch Intern Med 10 2003;163:317-323.

10 O'Meara ES, White M, Siscovick DS, et al: Hospitalization for pneumonia in the Cardiovascular Health Study: incidence, mortality, and influence on longer-term survival. J Am Geriatr Soc 2005;53:1108-1116.

11 Waterer GW, Kessler LA, Wunderink RG: Medium-term survival after hospitalization with community-acquired pneumonia. Am J Respir Crit Care Med 2004;169:910-914.

12 Brancati FL, Chow JW, Wagener MM, et al: Is pneumonia really the old man's friend? Two-year prognosis after community-acquired pneumonia. Lancet 1993;342:30-33.

13 Koivula I, Sten M, Makela PH: Prognosis after community-acquired pneumonia in the elderly: a population-based 12-year followup study. Arch Intern Med 1999;159:15501555.

14 Park DR, Sherbin VL, Goodman MS, et al: The etiology of community-acquired pneumonia at an urban public hospital: influence of human immunodeficiency virus infection and initial severity of illness. J Infect Dis 2001;184:268-277.

15 Goss CH, Rubenfeld GD, Park DR, et al: Cost and incidence of social comorbidities in lowrisk patients with community-acquired pneumonia admitted to a public hospital. Chest 2003;124:2148-2155.

16 Mundy LM, Auwaerter PG, Oldach D, et al: Community-acquired pneumonia: impact of immune status. Am J Respir Crit Care Med 1995;152:1309-1315.
17 Fine MJ, Auble TE, Yealy DM, et al: A prediction rule to identify low-risk patients with community-acquired pneumonia. N Engl J Med 1997;336:243-250.

18 Fang GD, Fine M, Orloff J, et al: New and emerging etiologies for community-acquired pneumonia with implications for therapy. A prospective multicenter study of 359 cases. Medicine (Baltimore) 1990;69: 307-316.

19 Washington State Death Data Files. Olympia, Washington State Department of Health, Center for Health Statistics, 2004.

20 SAS/STATA User's Guide, version 6. Cary, SAS Institute, 1991.

21 Collet D: Modeling Survival Data in Medical Research. New York, Chapman \& Hall, 1994.

22 Mickey RM, Greenland S: The impact of confounder selection criteria on effect estimation. Am J Epidemiol 1989;129:125-137.

23 Human Mortality Database. University of California, Berkeley (USA) and Max Planck Institute for Demographic Research (Germany). www.mortality.org (accessed January 2006).

24 Rothman KJ, Greenland S: Modern Epidemiology, ed 2. Philadelphia, Lippincott Williams \& Wilkins, 1998

25 Kuhn EM, Hartz AJ, Krakauer H, et al: The relationship of hospital ownership and teaching status to 30- and 180-day adjusted mortality rates. Med Care 1994;32:10981108 . 